\title{
Computação na Educação Básica no Brasil: o Estado da Arte
}

\section{Computer Science in the Brazilian Basic Education: State of the Art}

\author{
Adriana Bordini ${ }^{1,2}$ \\ Christiano Martino Otero Avila 1,3 \\ Yuri Weisshahn 1, 4 \\ Mônica Marques da Cunha 1, 5 \\ Simone André da Costa Cavalheiro 1, 6 \\ Luciana Foss 1, 7 \\ Marilton Sanchotene Aguiar ${ }^{1,8}$ \\ Renata Hax Sander Reiser ${ }^{1,} 9$
}

Data de submissão: 29/04/2016, Data de aceite: 22/08/2016

\begin{abstract}
Resumo: Este artigo apresenta um levantamento de projetos na área do Pensamento Computacional, com foco no ensino fundamental e médio, que tiveram resultados publicados nos principais veículos de Informática na Educação no Brasil. Neste estudo foi identificado que diferentes estratégias têm sido adotadas para a introdução da Computação na Educação Básica, destacando-se: Algoritmos e Programação, Robótica, Jogos, Computação Desplugada, entre outras. Cada uma das estratégias foi descrita considerando os seguintes aspectos: os conceitos da ciência da computação que têm sido abordados, as ferramentas utilizadas, a forma com que a estratégia tem abordado a interdisciplinaridade, a colaboração e/ou a comunicação. Ademais, uma descrição estatística dos trabalhos é realizada, destacando as instituições envolvidas nos projetos, o público alvo, as abordagens e ferramentas utilizadas. O objetivo deste relato é fornecer uma visão geral do que já foi alcançado na área no Brasil.
\end{abstract}

Palavras-chave: computação, educação básica, pensamento computacional, Brasil \footnotetext{
Grande Do Sul, Brasil.

2 adriana.bordinieinf.ufpel.edu.br\}

3 \{cm.avila@inf.ufpel.edu.br\}

4 \{yrweisshaln@inf.ufpel.edu.br

5 \{mmmd. cunha@inf.ufpel. edu.br\}

${ }^{6}$ \{simone. costa@inf.ufpel.edu.br\}

${ }^{7}$ \{lfosseinf.ufpel.edu.br\}

${ }^{8}$ \{mariltoneinf.ufpel.edu.br\}

${ }^{9}$ \{reisereinf.ufpel.edu.br\}
}

${ }^{1}$ CDTec/Computação, UFPel, Universidade Federal de Pelotas - Rua Gomes Carneiro, 1 - 96010-610 - Pelotas, Rio 


\begin{abstract}
This paper presents a report on projects in the area of Computational Thinking, focusing on primary and secondary schools, that were published in the main conferences on informatics and education in Brazil. This study identified that different strategies have been applied for the introduction of computing in basic education, particularly: Algorithms and Programming, Robotics, Games, Unplugged, among others. Each one of the strategies has been described considering the following aspects: the concepts of computer science that have been addressed, the adopted tools, the way in which the strategy has handled interdisciplinary, collaboration and/or communication. Also, a statistical description of the work is carried out, highlighting the institutions involved in the projects, the target audience, approaches and tools used. The goal of this report is to give an overview on what was achieved in the area in Brazil.
\end{abstract}

Keywords: computing, basic education, computational thinking, Brazil

\title{
1 Introdução
}

Pensamento Computacional (PC), do inglês Computational Thinking, pode ser entendido como um conjunto de técnicas que utiliza conceitos da Computação para solucionar problemas. Como proposto em [1], o PC considera a compreensão do comportamento humano, mas principalmente introduz uma maneira de raciocinar (de um Cientista da Computação) que inclui múltiplos níveis de abstração. Sua aplicabilidade nos mais diversos campos do conhecimento o torna uma habilidade fundamental para todas as pessoas, não apenas para Cientistas da Computação, despontando como um requisito elementar para a formação básica dos profissionais de todas as áreas nos próximos anos.

A busca em disseminar na prática o PC visa promover habilidades que auxiliem tanto para a solução de problemas do dia a dia como para a solução de problemas mais complexos da sociedade. Nos Estados Unidos e no Canadá, por exemplo, a Computer Science Teachers Association (CSTA) e a International Society for Tecnology in Education (ISTE) com o apoio da National Science Foundation (NSF) criaram o Computational Thinking in K-12 Education - Leadership Toolkit (CT-Toolkit) [2], que descreve as principais habilidades a serem desenvolvidas com a introdução do PC na educação básica.

Diversos projetos, direta ou indiretamente, já disseminam o PC na Educação Básica no Brasil. Alguns com objetivos claros de desenvolver habilidades e atitudes consideradas essenciais dentro do PC, outros, indiretamente, introduzindo conceitos de Computação. Este trabalho estende o estudo apresentado em [3], fornecendo um panorama mais geral e incluindo uma síntese dos resultados publicados na área com foco na Educação Básica no Brasil, restrita aos últimos seis anos. Assim, incluiu-se duas relevantes contribuições:

- uma síntese de mais de 60 artigos com resultados publicados na área, estabelecendo 
quais as estratégias que têm sido utilizadas para a disseminação deste processo de solução de problemas, quais os conceitos da computação que têm sido introduzidos, com quais ferramentas e como estes conceitos têm sido trabalhados de forma interdisciplinar ou colaborativa;

- uma descrição de alguns dados estatísticos do mapeamento realizado, destacando as instituições envolvidas nos projetos, o público alvo, as abordagens e ferramentas utilizadas, assim como a região em que os projetos foram desenvolvidos e os veículos em que seus resultados foram publicados.

O foco deste levantamento são artigos com projetos na área do PC que envolvam, tanto de forma lúdica como também de forma prática, o ensino de fundamentos da ciência da computação na Educação Básica do Brasil. A Educação Básica compreende os nove anos do Ensino Fundamental mais os três anos do Ensino Médio. A busca considerou artigos contendo resultados publicados nos principais veículos de Informática na Educação no Brasil.

$\mathrm{O}$ restante do artigo esta organizado como segue. A Seção 2 apresenta uma breve introdução ao Pensamento Computacional. Na Seção 3, apresenta-se a metodologia utilizada no mapeamento realizado e uma síntese de como o Pensamento Computacional está inserido na Educação Básica no Brasil. Na Seção 4, é descrita uma análise quantitativa do levantamento realizado. E concluindo, na Seção 5 , são apresentadas as considerações finais.

\section{Pensamento Computacional}

Pensamento Computacional é um processo de resolução de problemas, que inclui (mas não está limitado) as seguintes habilidades [1]: formulação de problemas de uma forma que seja possível usar um computador e outras ferramentas para ajudar a resolvê-los; organização lógica e análise de dados; representação de dados através de abstrações, como modelos e simulações; soluções de automação através do pensamento algorítmico (como uma série de passos ordenados); identificação, análise e implementação de soluções possíveis com o objetivo de alcançar a combinação mais eficiente e eficaz das medidas e recursos; generalização e transferência desse processo de resolução para problemas variados.

Essas habilidades são apoiadas e reforçadas por uma série de disposições ou atitudes que são dimensões essenciais do PC. Estas disposições ou atitudes incluem: a confiança em lidar com a complexidade; a persistência em trabalhar com problemas difíceis; a tolerância para a ambiguidade; a capacidade de lidar com os problemas em aberto; a capacidade de comunicar e trabalhar com outros para atingir um objetivo ou solução comum [2].

O PC pode ser colocado como uma das habilidades intelectuais básicas de um ser humano, comparada a ler, escrever, falar e fazer operações aritméticas [1]. Habilidades estas 
que servem para descrever e explicar situações complexas. Nesta linha de raciocínio, o PC é mais uma linguagem (como a linguagem escrita e a matemática) que pode ser utilizada para as pessoas falarem (descreverem) sobre o Universo e seus processos complexos.

\section{Pensamento Computacional na Educação Básica no Brasil}

O levantamento de projetos na área do Pensamento Computacional com o foco na Educação Básica no Brasil considerou a pesquisa em artigos publicados em periódicos e anais de conferências brasileiros, publicados nos últimos 6 anos, abrangendo o período de 2010 a 2015. Os veículos analisados foram: SBIE (Simpósio Brasileiro de Informática na Educação), WIE (Workshop de Informática na Escola), WAlgProg (Workshop de Ensino em Pensamento Computacional, Algoritmos e Programação), RBIE (Revista Brasileira de Informática na Educação), WEI (Workshop sobre Educação em Computação), WEIT (Workshop Escola de Informática Teórica) e CTD-IE (Concurso de TCC, Dissertações e Teses) do CBIE (Congresso Brasileiro de Informática na Educação).

A pesquisa direcionou-se principalmente a trabalhos que integrassem computação e educação básica mas com alguma forma de interface com o PC, seja pelas habilidades ou pelas atitudes trabalhadas. Inicialmente foi realizada uma pré-seleção considerando artigos que remetessem ao tema ciência da computação e educação básica, a qual englobou 103 artigos. Desta pré-seleção, optou-se por incluir no mapeamento apenas os trabalhos que tivessem resultados de aplicações práticas a estudantes do ensino básico, totalizando 62 publicações.

Dentre os trabalhos analisados, observou-se diferentes estratégias aplicadas na introdução da computação no ensino básico, das quais destacam-se: algoritmos e programação, robótica, jogos, computação desplugada, dentre outras (teatro/música e ensino híbrido).

O mapeamento do estudo realizado foi organizado a partir destas estratégias e, em cada uma delas, considerou-se quatro aspectos: os conceitos da ciência da computação que têm sido abordados, as ferramentas utilizadas, a relação com outras disciplinas e a forma com que a estratégia tem desenvolvido (se for o caso) a colaboração e/ou a comunicação.

\subsection{Estratégia Algoritmos e Programação}

Dentre os trabalhos considerados, 23 artigos adotam a estratégia de utilizar Algoritmos e Programação (AeP) para introduzir conceitos da computação. É considerada a mais popular e envolve abstração, automação, raciocínio lógico e algorítmico.

Os artigos identificados na estratégia de AeP podem ser classificados em 3 grupos: os que relatam experiência de ensino na área, os que propõem definir, aplicar e/ou avaliar estratégias de ensino/aprendizagem e os que avaliam o impacto do ensino de programação 
em outras áreas. As principais motivações destacadas nestes projetos foram: atrair jovens para a área da Computação, incentivar a autonomia tecnológica, incentivar a participação nas olimpíadas de programação, desenvolver habilidades do pensamento computacional, avaliar o impacto do ensino de programação no raciocínio lógico, complementar a formação e diminuir a evasão dos cursos de Licenciatura em Computação.

Identificou-se que os projetos utilizaram diferentes abordagens, as quais destacamse: introdução de conceitos básicos a partir da resolução de problemas lógicos ou desafios [4, 5, 6, 7, 8, 9, 10, 11, 12, 13, 14, 15, 16, 17, 18, 19, 20], ensino de linguagens estabelecidas [8, 9, 21, 11, 14, 13, 6, 7, 12], uso de ambientes visuais de programação [4, 10, 14, 17, 22, [18, 19, 23] ou proposta de novos ambientes [24].

3.1.1 Conceitos de Computação Dentre os relatos de experiências de ensino, a maioria focou em apresentar os conceitos básicos de algoritmos: tipos de dados básicos, variáveis, estruturas sequenciais, condicionais e de repetição [14, 9, 6, 7, 17, 19]. Alguns ainda incluíram variáveis compostas (vetores, matrizes ou registros) e modularização [11, 10, 20]. A grande maioria adotou ambientes de programação em blocos para introduzir conceitos de computação. Através da construção de scripts e animações os alunos também eram introduzidos ao conceito de depuração, testes, threads e paralelismo.

Alguns trabalhos focaram na introdução e na aplicação de estratégias metodológicas para o ensino de programação. Em [25, 23], a taxonomia de Bloom [26] foi aplicada como abordagem pedagógica para o processo de ensino/aprendizagem de AeP. Em [15, 23], Scaico et al. consideram a abordagem de ensino orientado ao design (proposta do ambiente Scratch) e, em [16], estes autores propõem a integração de cursos, gincanas e o ensino de programação como metodologia de sistematizar o ensino de Computação. Por sua vez, [14] relata que a adoção de cursos preliminares de programação por demonstração (associados ao uso da ferramenta StageCast Creator ${ }^{10}$ pode impactar no aprendizado posterior de lógica de programação. Já Torezani et al. [24] propõe o desenvolvimento de um ambiente web para auxiliar crianças na aprendizagem de conceitos iniciais de programação. Por fim, em [27] são investigadas possíveis contribuições de treinamento de lógica computacional, utilizando a linguagem Scratch, para o desenvolvimento do raciocínio lógico, identificando mudanças na fisiologia cerebral pré- e pós- treinamento de lógica computacional.

3.1.2 Uso de Ferramentas O desenvolvimento de jogos sem a necessidade de implementar código foi usado em [14], no ensino de lógica de programação. Cada estudante utilizou um computador individualmente para aprender a usar e criar jogos na ferramenta StageCast Creator, que é um software educacional voltado para o ensino da programação baseada em lógica.

$\overline{{ }^{10} \text { StageCast Creator - http: / / acypher. com/creator/ }}$ 
Outra ferramenta considerada foi o Scratch ${ }^{11}$, que também usa a técnica associada a exemplos. Este ambiente permite desenvolver programas de forma interativa e animada em um ambiente de programação bastante intuitivo. Em alguns trabalhos o Scratch foi utilizado para implementar algoritmos [8, 28, 16, 25, 27, 17, 18, 20], facilitando o processo de ensinoaprendizagem de algumas estruturas básicas de programação, incluindo o desenvolvimento de animações [28, 5, 15, 17, 22] e jogos [4, 19].

As linguagens $\mathrm{C}$ [8, 9], C++ [21, 20] e Python [11, 20] foram também empregadas na implementação de algoritmos e na resolução de problemas. Ainda na lógica de programação, alguns trabalhos usaram a linguagem de programação Portugo ${ }^{12}$ principalmente para o ensino dos primeiros algoritmos. Em alguns projetos, o Portugol foi usado na estruturação de algoritmos e desenvolvimento do raciocínio lógico [14, 13, 6, 7]. Em outro projeto [21], foi utilizado o BipIDE ${ }^{13}$ que permite que o estudante escreva um programa em Portugol e visualize o código equivalente na linguagem de montagem do processador BIP, podendo posteriormente realizar a execução das instruções, passo a passo, nos códigos fontes e na organização de um processador.

Sousa et al. [12] utilizaram a linguagem de programação Logd ${ }^{14}$ e o ambiente KTurtle ${ }^{15}$ para a criação de desenhos coloridos e na apresentação de mensagens em diferentes tamanhos, estilos e cores. Bezerra e Dias [29] também exploraram essa linguagem e esse ambiente para a realização de oficinas de programação de computadores.

O Logo é uma linguagem de programação desenvolvida no MIT (Massachussets Institute of Technology), criada para o ambiente escolar. O KTurtle é um ambiente que permite o uso dos comandos da linguagem Logo em português.

Em [10], o AppInventor ${ }^{16}$ foi utilizado para o desenvolvimento de aplicativos para quem não tem experiência em programação. AppInventor é uma plataforma de programação visual criada pela Google em parceria com o MIT, na qual se pode criar um aplicativo para Android ${ }^{17}$ utilizando blocos lógicos de maneira simples e intuitiva.

Em um dos artigos, desenvolveu-se o NewProg ${ }^{18}$ que é um ambiente web criado especificamente para apoiar o processo de aprendizagem de programação em crianças do ensino fundamental. As atividades propostas nesse ambiente priorizam o desenvolvimento de habilidade de resolução de problemas de vários níveis de complexidade. Em cada nível

\footnotetext{
${ }^{11}$ Scratch-https://scratch.mit.edu/

${ }^{12}$ Portugol -https://sourceforge.net/projects/portugolstudio/

${ }^{13}$ BipIDE - http://bipide.com.br/

${ }^{14}$ Logo - http://el.media.mit.edu/logo-foundation/what_is_logo/logo_programming. html

${ }^{15}$ KTurtle - https: //edu.kde.org/kturtle/

${ }^{16}$ AppInventor - http://appinventor.mit.edu/

${ }^{17}$ Android - http://www. android.com/

${ }^{18}$ NewProg - http://www. newprog.com.br/home.php
} 
de atividade são propostos problemas diferenciados, com o objetivo de ajudar as crianças na resolução de problemas complexos e estimular o raciocínio lógico [24].

3.1.3 Interdisciplinaridade Com a intenção de que os alunos desenvolvam o raciocínio para a resolução de problemas, trabalhou-se o raciocínio lógico-matemático juntamente com o raciocínio algorítmico [8]. A matemática também é cenário para alguns trabalhos [12, 6, 7, 18], que nas aulas de programação exploram exercícios com conteúdos da disciplina. Por exemplo na integração com a geometria, trabalham-se figuras geométricas, ângulos, operações com ângulos e reta; e no assunto de álgebra e funções, destaca-se a aplicação de expressões matemáticas, operações matemáticas e funções [12].

O Português também foi apontado como um dos conteúdos importantes para a construção de algoritmos pela necessidade da interpretação textual [6, 7]. Em [22], as animações desenvolvidas pelos alunos no computador, envolveram além da computação, mais duas disciplinas Português-Literatura e Artes. Anteriormente às aulas de computação, a história "Chapeuzinho Vermelho" foi abordada através da leitura e discussão nas aulas de PortuguêsLiteratura e após os alunos se expressaram por meio de desenhos dos personagens, nas aulas de Artes.

E ainda em [17], alunas do ensino médio desenvolveram animações para ajudar no aprendizado de conteúdos específicos de Matemática, Física e Química, sendo, respectivamente, Trigonometria, Leis de Newton e Fenômenos.

3.1.4 Colaboração e Comunicação Em alguns projetos, a comunicação foi estimulada pela troca de informações em grupos de trabalho de alunos [8, 21], fazendo com que buscassem as respostas entre si, compartilhando conhecimento, para depois buscar ajuda do professor. Em [10], a convivência com profissionais do mercado de computação foi muito elogiada por todos participantes do curso. Concluiu-se que é importante investir em palestras e oportunidades de troca de experiências dos alunos com estes profissionais.

Os estudantes também foram estimulados a trabalhar com outras pessoas no processo criativo em [16] e na criação de projetos em [15]. Conforme destacado em [5, 25], o ato de trabalhar em pares, no computador, motivou a colaboração no ato de aprender e na proposição de soluções para os problemas levantados. Já o Projeto Jovem Hacker [4] incentivou o trabalho em grupo na construção e simulação de scripts.

A cooperação é também adotada como abordagem pedagógica em [24], onde inicialmente as atividades eram realizadas individualmente e, em segundo momento, elas eram realizadas com o auxílio de colegas. Em [22], observou-se um desconforto inicial dos alunos nos trabalhos em dupla, que depois foi superado. Após as primeiras etapas do projeto, ficou evidente que os alunos desejavam mostrar e compartilhar suas descobertas e resultados com os colegas e instrutores, motivando os alunos a ajudarem uns aos outros no contexto do grupo. 
O projeto Olimpíada de Programação de Computadores para Estudantes do Ensino Fundamental [18] criou olimpíadas entre escolas participantes do mesmo no município, envolvendo trabalho em equipes para a resolução de desafios. Esta metodologia promoveu a colaboração entre os membros de uma mesma equipe, estimulando a comunicação entre alunos de uma mesma escola, de anos diferentes e até de outras instituições.

\subsection{Estratégia Robótica}

A Robótica Pedagógica ou Robótica Educacional permite vivenciar, na prática, o resultado do que foi desenvolvido por meio da programação. Os dispositivos robóticos respondem a estímulos (entradas), que são captadas por sensores; e respondem (saídas) por meio de atuadores (motores, rodas, engrenagens). A captura das entradas e as saídas dos atuadores são realizadas por meio da programação. A estratégia engloba computação física, pois tratase de equipamentos de computação que interagem e respondem a realidade física (analógica) de um determinado ambiente, exigindo dos envolvidos uma relação física com montagem e funcionamento de objetos. Nesta seção, 11 textos reportam a robótica como um facilidador na aprendizagem de conceitos relacionados, mesmo que não explicitamente, ao PC.

3.2.1 Conceitos de Computação Em relação à programação, a robótica permite que o aprendiz verifique, fisicamente, aquilo que programou (ou tentou). A maioria dos artigos que foram selecionados nesta seção não detalhou quais conceitos (decisão/condicional, repetição, variáveis, etc.) pretendia trabalhar no projeto relatado. Em [30] os autores relatam que foram trabalhados os conceitos básicos relacionados às estruturas de controle de execução de um programa, sequencial, condicional e de repetição, além de conceitos de níveis intermediários como subprogramas. Um aspecto interessante da robótica educacional é a ludicidade. Alguns autores [30, 31] utilizaram a robótica com o objetivo de tornar o ensino da lógica de programação e o consequente estudo dos conceitos, o mais divertido, simples e interessante possível.

3.2.2 Uso de Ferramentas Em relação à utilização de programas (softwares) e kits, temse a adoção do kit de robótica Br_GOGO juntamente com um conjunto de softwares livres em [32] para implementar robótica pedagógica de baixo custo; e do kit de Robótica ROBO TX Explorer ${ }^{19}$ da empresa Fischertechnik que utiliza ambiente de programação chamado ROBO Pro em [30]. O kit de robótica Lego Mindstorms NXT foi utilizado nos projetos relatados em [33], [30], [34], [35] e [36]. Na programação dos robôs, além do ROBO Pro [30], tem-se a utilização do software que acompanha o kit de robótica da Lego, o NTX-G em [37] e [34]. O Robocode ${ }^{20}$ da AlphaWorks (uma divisão da IBM) é utilizado em [31]

${ }^{19}$ ROBO TX Explorer-http://www.fischertechnik.de/

${ }^{20}$ Robocode - http://robocode. sourceforge.net/ 
e permite trabalhar em um ambiente de simulação de batalha entre robôs programando em Java e o software Scratch, permitindo desenvolver programação para controlar uma interface eletrônica conectando motores, sensores, luzes, dentre outros componentes do robô.

3.2.3 Interdisciplinaridade A interdisciplinaridade emerge quando se fala em robótica educacional. Em [32] são apresentados experimentos na área de física com os assuntos Movimento Retilíneo Uniforme (MRU e Movimento Retilíneo Variado (MRV), onde um carrinho (robô) é programado para passar sobre linhas pretas com velocidades constante e variada, respectivamente, detectando e registrando cada passagem. Após coletar os dados, a partir dos experimentos, os alunos foram instigados a responder questões sobre MRV/MRU, que envolviam gráficos e interpretação do coeficiente angular dos gráficos, dentre outras questões.

Em um outro exemplo de aplicação de robótica [38], os autores relatam um projeto de um estacionamento automatizado, realizado com alunos do $1^{0}$ ano do ensino médio, trabalhando conceitos de física e matemática (geometria plana - razão, proporção, escala). Também na área de matemática, em [34], descreve-se um projeto de um robô que anda sobre uma superfície plana para que sejam realizados cálculos, envolvendo rotação das rodas e do motor, o diâmetro das rodas e a distância percorrida. O computador educacional Classmate, distribuído pelo governo federal via projeto $\mathrm{UCA}^{21}$, foi utilizado em [39] como o cérebro robótico de experiência que procura desenvolver conceitos como tempo, espaço, distância, velocidade e potência. Similarmente, em [36], os autores relatam a estratégia de utilizar a ludicidade por meio de um desafio de Caça ao Tesouro, onde os alunos programam um robô para chegar em um determinado local seguindo um trajeto que envolve cálculos de trajetórias com retas e curvas.

3.2.4 Colaboração e Comunicação Nos artigos analisados, que envolvem a abordagem robótica como um instrumento para a disseminação de conceitos relacionados ao PC, em sua grande maioria, não foi possível identificar como ou se foram incentivados processos de colaboração e comunicação entre os participantes do projeto. Em [30] é relatado que são formadas duplas para a montagem dos robôs, o que permitiria a colaboração entre os participantes. A partir de algumas fotos que estão colocadas no artigo também é possível inferir que os participantes foram incentivados a colaborar durante a realização do processo em função de que as peças estão colocadas em uma mesa com os participantes sentados ao seu redor, porém não há um relato específico em relação ao incentivo para um processo colaborativo mais estruturado.

Processos colaborativos em [37], baseados na criação de grupos de participantes, estimulam como tarefas: $a$ ) familiarização com o ambiente de programação do Mindstorms; $b$ ) a apresentação dos comandos do software; $c$ ) a apresentação dos componentes e sensores dos

$\overline{{ }^{21} \text { Projeto UCA - http://goo.gl/ve45us }}$ 
robôs; $d$ ) a montagem dos robôs; $e$ ) a criação de algoritmos para solução dos problemas e desafios propostos. Da mesma forma em [32] são formados grupos para desenvolver as atividades do ambiente de Robótica Pedagógica (design, construção, automação-programação e controle do dispositivo robótico). A formação de grupos, como incentivo a um processo colaborativo, é também descrita em [36], onde os autores explicam que foram organizados 5 grupos de 4 alunos e para cada grupo foi fornecido um kit. O objetivo era deixá-los à vontade para montar qualquer tipo de robô.

\subsection{Estratégia Jogos Digitais}

Diversos são os formatos relatados em 16 artigos na utilização dos jogos digitais para ensino-aprendizagem de conceitos relacionados ao PC, porém observa-se que, em sua grande maioria, os correspondentes projetos utilizam como estratégia o desenvolvimento ou criação de jogos para proporcionar o aprendizado, em oposição à utilização de jogos especializados que proporcionam o aprendizado de programação. E deste total, apenas quatro relataram atividades relacionadas à utilização de um jogo para ensino-aprendizagem de conceitos relacionados ao PC. Os demais trabalhos relatam a utilização de ambientes para a criação, por parte dos aprendizes, de novos jogos, mesmo que simples ou de baixa complexidade, como forma prioritária para ensino-aprendizagem dos conceitos.

3.3.1 Conceitos de Computação Os conceitos básicos de programação considerados na abordagem de jogos digitais são apresentados nos trabalhos descritos a seguir. Em [40], os alunos foram incentivados a resolver desafios, inicialmente utilizando as estruturas de sequência, condicional e repetição e, num segundo momento, envolvendo funções e recursão. Neste mesmo projeto os autores adotam ferramentas que permitem utilizar trechos de códigos prédefinidos, incluindo recursos como strings, arrays e objetos. Ainda com foco na ludicidade, em [4] foram aplicadas dinâmicas ao ar livre e trabalhados conceitos como variáveis, paralelismo na execução de threads e condicionais.

Nos trabalhos relatados em [41, 42, 23, 43, 44], os alunos foram estimulados a criar seus próprios jogos envolvendo noções elementares de programação (variáveis, operadores lógicos e relacionais, estruturas de sequência, de seleção e de repetição). Em [41], atividades propostas também incluíram a leitura de sensores. Já em [43] ênfase também foi dada ao conceito de paralelismo.

3.3.2 Uso de Ferramentas Dentre os artigos que se enquadram nesta estratégia, foi possível observar que em [45], foi utilizado o jogo Lightbot ${ }^{22}$ com crianças entre 5 e 13 anos. O jogo foi testado e a avaliação, segundo os autores, "mostrou que a ferramenta tem potencial para a aprendizagem por descoberta apoiada por preceitos construtivistas e construcionis-

${ }^{22}$ Lightbot - http://lightbot.com/ 
tas porém foram constatadas dificuldades em relação a não permitir a total autonomia das crianças sendo necessário a presença de um mediador para guiá-las".

No Clube de Programação proposto em [40], mais três jogos são considerados, além do Lightbot. O Labirinto, disponível no site Code.org, em que é necessário usar blocos de sequência, condicionais e repetição para guiar um personagem até o final de um labirinto. O Code Combat ${ }^{23}$, que permite exercitar conceitos de estruturas dados e de controle. E o Scratch, viabilizando a construção de uma animação que simula um jogo de ping-pong.

O ambiente de desenvolvimento Scratch, desenvolvido no MIT, é preferencialmente citado quando se trata do desenvolvimento de jogos para ensino-aprendizagem de conceitos relacionados ao PC, sendo utilizado em nove dos 16 projetos analisados. Dentre outras ferramentas citadas nos artigos analisados, tem-se a linguagem de programação Python em conjunto com a biblioteca de jogos multiplataforma PyGame ${ }^{24}$ utilizada em [46]; a Stency ${ }^{25}$ ([47, 48]) que, similarmente ao Scratch, possibilita a criação de jogos para dispositivos móveis, web e desktop através do encaixe de blocos de comando; o NoBug's Snack Bar ${ }^{26}$ ([49]), jogo que também utiliza a lógica visual do encaixe de blocos para programação; o GameMaker Studid ${ }^{27}([44])$, ferramenta proprietária, da empresa YoYo Games, que permite o desenvolvimento de jogos; e a StageCastCreator ([14]), que é uma linguagem de programação visual baseada no conceito de programação por demonstração, onde são criadas regras a partir de exemplos de ações que devem ser realizadas em determinadas situações.

3.3.3 Interdisciplinaridade Dentre os 16 trabalhos, em apenas dois projetos fica explícita a preocupação com a interdisciplinaridade na proposta de desenvolver noções básicas do PC. Em [50] é relatado o desenvolvimento de jogos digitais com desafios de matemática, onde os aprendizes são alunos do $5^{\circ}$ ano do ensino fundamental e os conteúdos matemáticos que serviram de temática para o desenvolvimento dos jogos foram abordados no trimestre em que o projeto estava ocorrendo. Segundo os autores, a criação de jogos digitais integrados com o currículo foi uma forma de motivar o estudo, aliando diversão e aprendizagem.

Também em [51], projeto que atua junto aos alunos do primeiro ano do ensino médio de uma escola pública, incentiva-se os estudantes a desenvolver um jogo educativo de matemática que envolvesse as quatro operações básicas, sugerindo cálculos aleatórios. No mesmo trabalho [51], relata-se o desenvolvimento de mais dois jogos: o "TreiENEM", que é um jogo de perguntas e respostas sobre conceitos de matemática, voltado para quem quer aprimorar seus conhecimentos e se preparar para as provas dos vestibulares, com questões extraídas do Exame Nacional do Ensino Médio (ENEM); e o R3cicl@!, que enfatiza a conscientização

${ }^{23}$ Code Combat - http://br. codecombat.com/

${ }^{24}$ Pygame - http://pygame.org/hifi.html

${ }^{25}$ Stencyl - http://www.stencyl.com/game/showcase/

${ }^{26}$ NoBug's Snack Bar - http://nobugssnackbar.dei.uc.pt/

${ }^{27}$ GameMaker - http://www.yoyogames.com/gamemaker 
para preservação do Meio Ambiente, especialmente no que diz respeito à compreensão de que é necessário reciclar o lixo não orgânico.

3.3.4 Colaboração e Comunicação Em relação a colaboração, poucos projetos trabalham, ou explicitam em seus relatos, atividades colaborativas que envolvam o PC e jogos digitais. Em [4], os autores descrevem o projeto Jovem Hacker que visa "auxiliar na formação de uma geração que seja autônoma tecnologicamente e se empodere dos rumos da nossa sociedade", enfatizando a importância da colaboração. Particularmente, destacam que atividades de socialização e a realização de dinâmicas que envolvem a simulação de conceitos de computação motivam os estudantes e parecem facilitar a aprendizagem dos conteúdos trabalhados.

Em [47] foi explorada a avaliação por pares, incluindo no desenvolvimento do trabalho etapas nas quais os estudantes avaliavam as atividades desenvolvidas por colegas, de acordo com critérios pré-estabelecidos. Segundo os autores, esta estratégia melhorou significativamente os resultados de aprendizagem dos estudantes e proporcionou um trabalho colaborativo, já que eles praticavam sua análise crítica e conseguiam melhorar os seus projetos com as avaliações recebidas .

Ainda com ênfase na colaboração, os trabalhos descritos em [50, 51] envolveram a criação e programação de jogos por meio de trabalhos em grupos. Embora esta estratégia tenha estimulado o trabalho colaborativo e interpessoal, a ferramenta utilizada não permitiu a implementação conjunta, o que foi considerado como um ponto negativo pelos autores.

\subsection{Estratégia Computação Desplugada}

A computação Desplugada é uma estratégia que visa ensinar os fundamentos da computação de forma lúdica, sem o uso de computadores e sem aprofundar detalhes técnicos. A abordagem pode ser aplicada para pessoas de todas as idades, desde o ensino fundamental até o ensino superior, com diferentes conhecimentos e experiências. Um dos objetivos é eliminar as barreiras técnicas e os equívocos sobre o que é realmente a computação. Segundo [52], os princípios da estratégia consistem em não utilizar computadores, ensinar ciência da computação, aprender fazendo, tornar o ensino divertido, não adotar nenhum equipamento especializado, desenvolver variações sobre aplicações das atividades, poder ser aplicada para qualquer pessoa, enfatizar a cooperação incluindo comunicação e solução de problemas, desenvolver atividades autossuficientes, ou seja, que possam ser usadas independentemente umas das outras e por fim, ser flexíveis com relação a erros, isto é, pequenos erros não devem impedir que os participantes entendam os fundamentos.

Foram incluídos nesse estudo 18 textos, os quais citam diferentes formas e experiências de metodologias envolvendo a técnica da Computação Desplugada. Dentre as motivações 
dos autores que adotam tal abordagem, destacam-se a introdução e disseminação do ensino de computação, o aumento da autonomia tecnológica e a complementação na formação de estudantes do curso de Licenciatura em Computação.

Embora algumas das atividades aplicadas e/ou propostas envolvam conteúdos interdisciplinares, nenhum dos trabalhos propôs efetivamente a integração com disciplinas curriculares dos anos trabalhados. Além disso, pela própria natureza da técnica, nenhum tipo de ferramenta tecnológica é utilizada nesta abordagem, apenas a construção e utilização de materiais didáticos concretos. Portanto, as subseções de Interdisciplinaridade e Ferramentas são omitidas nesta apresentação.

3.4.1 Conceitos de Computação Todos os trabalhos que utilizam esta abordagem aplicam atividades desplugadas para o ensino de computação. Enquanto diversos dos resultados [33, 28, 53, 15, 16, 52, 54, 55, 56, 57, 58, 44, 35, 59] provém de aplicações de atividades do livro Computer Science Unplugged [60], alguns trabalhos [61, 62, 63] propõe novas atividades para o ensino da Computação Desplugada. Para todas as atividades da Parte I do livro (para a qual existe uma versão traduzida para o português) já existem relatos de aplicação na educação básica. Segue a lista das atividades trabalhadas com os respectivos conteúdos envolvidos: Contando os Pontos (números binários), Colorindo com Números (representação de imagens), Você pode Repetir (compressão de texto), A Mágica de Virar as Cartas (detecção e correção de erros), Vinte Palpites (teoria da informação), Batalha Naval (algoritmos de busca), O Mais Leve e o Mais Pesado (algoritmos de ordenação), Seja o Mais Rápido (redes de ordenação), A Cidade Enlameada (árvores geradoras mínimas), O Jogo da Laranja (roteamento e bloqueio nas redes), Caça ao Tesouro (autômatos de estados finitos) e Seguindo Instruções (linguagens de programação).

Alguns trabalhos, além de atividades do livro, aplicaram atividades de diferentes fontes. Em [52], foi também aplicada a atividade Transmitindo uma Mensagem com Segurança para trabalhar o método da chave única. Santos et al. [62] utiliza a confecção de origamis e o jogo de tabuleiro RobotTurtle ${ }^{28}$ para introduzir o conceito de algoritmos.

Por fim, existem trabalhos que propõem atividades desplugadas. Arantes et al. [64] apresenta uma dinâmica ao ar livre para simulações de scripts e da execução de programas, trabalhando os conceitos de variáveis, concorrência na execução de threads e condicionais. Hinterholz et al. [61] descreve um conjunto de atividades para a introdução de fundamentos de banco de dados (introduzindo conceitos básicos como tabelas, dados, elementos de dados, relacionamento de tabelas por chave primária e chave estrangeira e sistemas de gerenciamento de banco de dados). Em [65] a atividade Algoritmos Desplugados introduz conceitos básicos de algoritmos. As atividades em [63] trabalham com coleta, análise e representação de dados (atividade inspirada no jogo Cara a Cara), algoritmos, procedimentos e simulação (atividade

${ }^{28}$ RobotTurtles - http://www.robotturtles.com 
que consiste na Caça ao Tesouro) e decomposição de problemas, paralelismo e automação (atividade de Organização de uma Festa).

3.4.2 Colaboração e Comunicação No contexto da Computação Desplugada, mais uma vez, observa-se que pouco é explorado o trabalho colaborativo e comunicação. Usualmente as propostas preveem atividades em duplas ou em grupos, mas pouco é planejado de maneira a fomentar a habilidade de se comunicar e trabalhar com outros para atingir um objetivo ou uma solução comum. Ressalta-se a dinâmica proposta em [64], a qual envolve grupos de estudantes na simulação de scripts e programas, promovendo através do trabalho colaborativo uma visão clara entre o papel dos atores e de suas interações na execução de um programa.

\subsection{Outras Estratégias}

Em 3 dos textos analisados são usadas estratégias alternativas para a introdução da Computação na Educação Básica. Uma delas consiste na integração do teatro, música ou dança com o ensino de computação, tornando as atividades mais atrativas para os alunos, já que se utilizam do entretenimento para isso. Particularmente, o trabalho desenvolvido em [33] motivou o uso da música pelo seu potencial em despertar emoções, de atuar no desenvolvimento de processos cognitivos, como o raciocínio e a criatividade, além de incentivar a cooperação e socialização. E, a atividade interativa de apreender computação através do Teatro [52] consiste em um processo criativo, dinâmico e prazeroso.

Numa abordagem hibrida, em 2 dos projetos analisados, o ambiente de aprendizagem organiza-se sobre dois pilares essenciais: aprendizagem em sala de aula e aprendizagem online (que muitas vezes ocorre à distância). Na abordagem online, o ensino se caracteriza pela conexão de diversos tipos de mídias eletrônicas e tecnologias da informação e comunicação ligados a educação, caracterizada como experiência de ensino via suporte computacional. Esta abordagem enfatiza as contribuições oferecidas pelos artefatos tecnológicos digitais, que envolve tecnologias baseadas na web (por exemplo, sala de aula virtual ao vivo, aprendizagem adaptativa e/ou colaborativa, streaming de vídeo, áudio e texto), ferramentas tecnológicas (por exemplo, lousas digitais, computadores, câmeras) e o modelo tradicional, a fim de atingir um objetivo educacional. Quanto a metodologia, são mescladas diversas abordagens pedagógicas (por exemplo, aprendizagem significativa, behaviorismo, construcionismo, construtivismo, cognitivismo) resultando em aprendizagem com ou sem tecnologias de ensino [66].

3.5.1 Conceitos de Computação Em [33], uma oficina foi desenvolvida, com o objetivo de introduzir o conceito de algoritmos através da produção de ritmos. O instrumento escolhido foi o pandeiro, que compreende quatro golpes fundamentais denominados: open tone, finger, heel e slap, tratados como o conjunto de instruções. A execução desses golpes no 
pandeiro produzia diferentes sons, e sequências distintas destes golpes produzia ritmos diferentes. Em [52], a metodologia utilizada foi a de apresentar os conteúdos de Computação através de uma peça teatral lúdica, interativa e dinâmica. A peça teatral consistia na apresentação de atividades do livro Computer Science Unplugged [60] que tratam dos seguintes conteúdos da área de computação: detecção de erros, criptografia (método da chave única), números binários (conversão da base 2 para base 10), representação de imagens, bloqueio nas redes (resolução com sistemas colaborativos) e algoritmos de ordenação (BubbleSort e InsertionSort). Já em [67], foram trabalhados os diferentes algoritmos de ordenação através da Dança. Os algoritmos simulados foram: BubbleSort, QuickSort e SelectionSort.

$\mathrm{Na}$ abordagem híbrida, os autores abordaram conceitos de algoritmos e programação (comandos de entrada e saída, variáveis, estrutura de controle, estrutura de dados e funções) [68, 9], conceitos básicos do que é computação e do que é informação, pensamento algorítmico através de sequência de passos e resolução de problemas lógicos [68].

3.5.2 Uso de Ferramentas Nos trabalhos que adotaram o ensino híbrido, destaca-se em [9] o uso do Ambiente Virtual de Aprendizagem Moodle para as atividades não presenciais. $\mathrm{O}$ ambiente contém uma sala de aula virtual onde o aluno tem a possibilidade de acompanhar ou desenvolver as atividades propostas pelo professor. Ele foi utilizado para discutir dúvidas, divulgar materiais extras e permitir a comunicação direta entre alunos e professores fora da sala de aula. Em [68] as atividades online foram caracterizadas pelo uso da ferramenta AppInventor para a criação de aplicativos Android.

3.5.3 Interdisciplinaridade Em [67], foi desenvolvida uma oficina que ligava os conceitos da Computação às disciplinas cotidianas do Ensino Básico. A primeira intervenção foi realizada na disciplina de Artes, cujo conteúdo curricular a ser trabalhado foi a dança regional. O objetivo da intervenção foi ensinar como funciona a ordenação dos dados no computador, por meio da dança. Em analogia ao vídeo da dança folclórica húngara, realizada junto ao projeto AlgoRythmics da Sapientia University da Romênia, as danças selecionadas em [67] auxiliaram na organização de uma sequência ordenada e crescente, e teve como temas musicais o Forró/Xote e o Samba Regional.

3.5.4 Colaboração e Comunicação Em [68] a comunicação e colaboração foi explorada tanto no desenvolvimento do pensamento algorítmico, onde os alunos definiam sequências de passos para a construção de desenhos que eram executadas por colegas, como no desenvolvimento conjunto de aplicativos móveis. 


\section{Descrição Estatística do Levantamento Realizado}

Esta seção tem o intuito de apresentar uma descrição sobre alguns dados estatísticos coletados a partir dos 62 artigos que foram estudados.

\subsection{Público-alvo dos Projetos}

A partir da análise dos artigos foi possível identificar, conforme a Figura 1, que os projetos estão bem divididos entre o ensino fundamental e médio com exatamente $42 \%$ para cada um dos níveis. Alguns projetos relatados possuem ações que atingem ou mesclam públicos nos dois níveis. Neste caso classificamos, para fins de público-alvo, em "Ensino Fundamental e Médio".

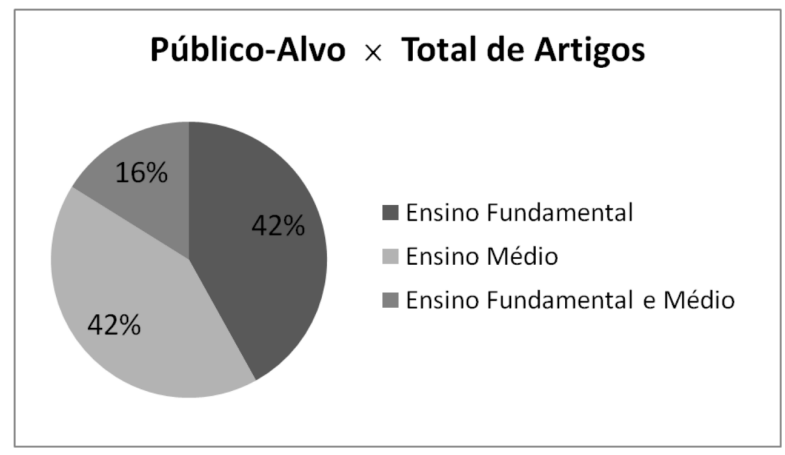

Figura 1: Total de artigos por público-alvo.

\subsection{Abordagens}

Considerando as estratégias para disseminação do PC descritas na Seção 3 e em acordância com o gráfico apresentado na Figura 2 reitera-se que a abordagem Algoritmos e Programação é a mais utilizada, seguida da abordagem Computação Desplugada.

É importante destacar que alguns projetos adotam mais de uma abordagem como, por exemplo, Computação Desplugada e Jogos Digitais. Neste caso, em uma oficina são abordados os conteúdos e atividades da Computação Desplugada em um módulo e em outro são aplicados jogos para apresentar seus conceitos. Tais casos foram contabilizados nas duas abordagens na Figura 2 e, em função disso, a soma do total de artigos por abordagem chega a 75 , ultrapassando o número total de artigos avaliados (62). 


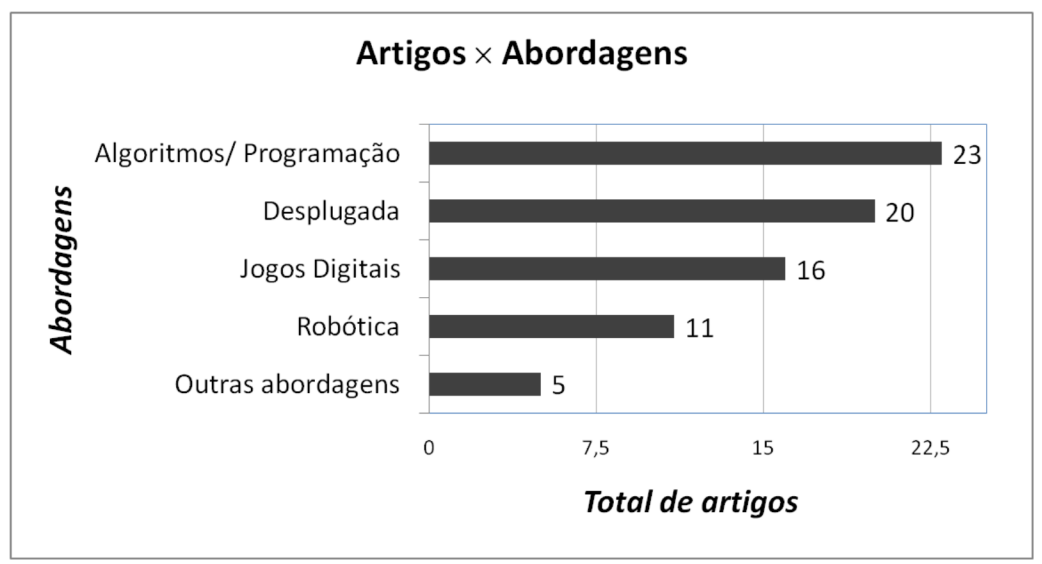

Figura 2: Total de artigos por abordagem.

\subsection{Eventos}

Em relação aos eventos, apresentados na Tabela 1, constata-se que ainda é pouco significativa a presença da área de PC na maioria dos eventos. Apenas $4 \%$ dos artigos publicados nestes eventos, entre 2010 e 2015, abordam esta área. No maior evento considerado, o SBIE, apenas $0,5 \%$ dos artigos publicados abordavam o PC. $\mathrm{O}$ evento que se destacou com um percentual de $23 \%$ de artigos sobre PC, foi o $W$ AlgProg. Isso também reflete a questão da abordagem de Algoritmos e Programação ser a mais utilizada nos trabalhos que envolvem o tema.

Considerando-se somente os artigos analisados (62), o WIE e o WEI são os dois eventos que mais publicaram trabalhos que contribuíram para a disseminação do PC na Educação Básica no referido período, totalizando 46 artigos ou aproximadamente $74 \%$ dos artigos.

\subsection{Ano de Publicação}

Ao observar o total de artigos selecionados neste estudo, é possível verificar que o tema, objeto deste trabalho, obteve maior interesse dos pesquisadores brasileiros a partir do ano de 2013 (veja Figura 3), já que nos últimos três anos (2013-2015) acumulam-se mais de $85 \%$ dos artigos estudados, sendo que $56 \%$ do total foi no ano de 2015 . Porém, é importante ressaltar que o foco deste levantamento foi analisar os relatos (estudos de caso) sobre PC no Brasil com aplicação direta na Educação Básica. Isto fez com que diversos artigos fossem desconsiderados, por tratarem de outros públicos (Ensino Técnico, Ensino Superior, etc.) ou por trazerem relatos de ferramentas ou métodos do PC sem aplicação direta no Ensino Básico. 
Tabela 1: Classificação por evento dos artigos analisados.

\begin{tabular}{lcc}
\hline Eventos Brasileiros & Total de Artigos & Artigos Analisados \\
\hline WIE & 368 & 25 \\
WEI & 226 & 21 \\
WAlgProg & 30 & 7 \\
SBIE & 812 & 4 \\
RBIE & 172 & 3 \\
CTD-IE (2015) & 17 & 1 \\
WEIT & 82 & 1 \\
\hline TOTAIS & $\mathbf{1 7 0 7}$ & $\mathbf{6 2}$ \\
\hline
\end{tabular}

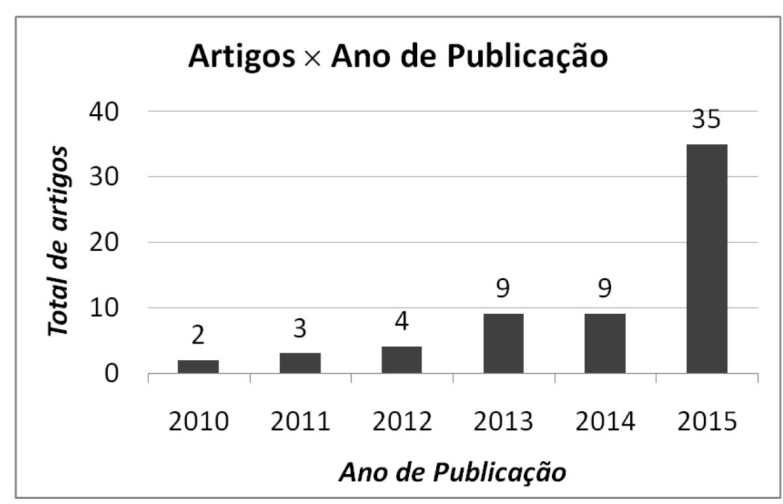

Figura 3: Total de artigos por ano de publicação.

\subsection{Ferramentas}

Esta seção apresenta alguns dados que foram extraídos dos artigos analisados referentes às ferramentas que foram utilizadas para a implementação de projetos relacionados ao PC. $\mathrm{Na}$ Tabela 2 estão relacionadas as ferramentas que foram adotadas em dois ou mais projetos. É possível visualizar que o ambiente de programação Scratch foi utilizado em mais de $40 \%$ dos projetos. É importante ressaltar que, de forma similar às abordagens, os projetos relatados nos artigos que foram analisados, em geral, utilizam mais de uma ferramenta. Os percentuais de utilização foram calculados baseados no total de 62 artigos. Outras 19 ferramentas, que também foram utilizadas nos artigos estudados, foram usadas em apenas um artigo e não foram adicionadas à tabela. Alguns detalhes, em relação às ferramentas utilizadas pelos projetos analisados, podem ser conhecidos na Seção 3 deste artigo. 
Tabela 2: Ferramentas usadas por mais de um projeto.

\begin{tabular}{lcc}
\hline Ferramentas & $\begin{array}{c}\text { Total de } \\
\text { artigos }\end{array}$ & $\%$ \\
\hline Scratch & 25 & 40,3 \\
Desplugada & 18 & 29,0 \\
Portugol & 5 & 8,1 \\
Kit Robótica Lego & 5 & 8,1 \\
Python & 4 & 6,5 \\
Lightbot & 3 & 4,8 \\
LinguagemC & 2 & 3,2 \\
Logo & 2 & 3,2 \\
Kturtle & 2 & 3,2 \\
App Inventor & 2 & 3,2 \\
Hora do Código & 2 & 3,2 \\
Stencyl & 2 & 3,2 \\
Code Combat & 2 & 3,2 \\
C++ & 2 & 3,2 \\
\hline
\end{tabular}

\subsection{Instituições}

A Tabela 3 apresenta a distribuição das instituições que produziram os artigos analisados. Os percentuais foram calculados sempre considerando o total de 62 artigos. Alguns artigos possuem mais de uma instituição envolvida na sua escrita, assim a contabilização destes artigos é dividida entre as instituições participantes. Por exemplo, um artigo que foi escrito por autores de três diferentes instituições, contabiliza 0,33 para cada uma. Analisando a tabela, pode-se observar que das 36 instituições, 16 estão relacionados com menos de um artigo, isto é, apenas possuem artigos compartilhados. Além disso, 7 instituições possuem apenas um artigo entre os analisados. Uma grande concentração dos artigos está nas primeiras cinco instituições (aproximadamente $45 \%$ ).

\subsection{Estados das instituições}

No gráfico da Figura 4 é possível verificar que a produção de artigos sobre pensamento computacional (cases com aplicação no ensino básico) estão concentrados em apenas 11 estados da federação. Das 27 unidades (estados + DF), 16 não possuem trabalhos dentro do foco analisado neste estudo. A região nordeste se destaca com $33,9 \%$ dos trabalhos analisados. 
Tabela 3: Envolvimento das instituições na produção dos artigos.

\begin{tabular}{|c|c|c|}
\hline Instituiçõos & $\begin{array}{l}\text { Número de } \\
\text { artigos }\end{array}$ & $\%$ \\
\hline Universidade Federal da Paraíba (UFPB) & 9 & 14,52 \\
\hline Universidade Federal Rural da Amazônia (UFRA) & 6 & 9,68 \\
\hline Universidade de Pernambuco (UPE) & 4,5 & 7,26 \\
\hline Universidade Estadual de Campinas (UNICAMP) & 4,33 & 6,99 \\
\hline Universidade Federal de Pelotas (UFPEL) & 4 & 6,45 \\
\hline Universidade Federal da Bahia (UFBA) & 3 & 4,84 \\
\hline Universidade Federal Rural de Pernambuco (UFRPE) & 3 & 4,84 \\
\hline Universidade Federal de Pernambuco (UFPE) & 2,5 & 4,03 \\
\hline Universidade Federal de Uberlândia (UFU) & 2,5 & 4,03 \\
\hline Universidade do Vale do Itajaí (UNIVALI) & 2 & 3,23 \\
\hline Universidade Estadual de Feira de Santana (UEFS ) & 2 & 3,23 \\
\hline Universidade Federal do Espírito Santo (UFES) & 2 & 3,23 \\
\hline Universidade Federal do Rio Grande (FURG) & 2 & 3,23 \\
\hline Universidade de Santa Cruz do Sul (UNISC) & 1 & 1,61 \\
\hline Universidade do Estado de Santa Catarina (UDESC) & 1 & 1,61 \\
\hline Universidade Estadual da Paraíba (UEPB) & 1 & 1,61 \\
\hline Universidade Federal de Santa Catarina (UFSC) & 1 & 1,61 \\
\hline Universidade Federal de Santa Maria (UFSM) & 1 & 1,61 \\
\hline Universidade Federal de Sergipe (UFS) & 1 & 1,61 \\
\hline Universidade Federal do Amazonas (UFAM) & 1 & 1,61 \\
\hline Faculdade Meridional (IMED-Passo Fundo) & 0,83 & 1,34 \\
\hline Universidade de Passo Fundo (UPF) & 0,83 & 1,34 \\
\hline Universidade Federal do Rio de Janeiro (UFRJ) & 0,83 & 1,34 \\
\hline Centro Educacional Pioneiro & 0,5 & 0,81 \\
\hline Faculdade de Tecnologia de Jahu (FATEC-Jahu) & 0,5 & 0,81 \\
\hline Faculdade de Tecnologia do Estado de São Paulo (FATEC) & 0,5 & 0,81 \\
\hline Instituto Federal Baiano (IFBaiano-Senhor do Bonfim) & 0,5 & 0,81 \\
\hline Universidade de São Paulo (USP) & 0,5 & 0,81 \\
\hline Universidade do Estado da Bahia (UNEB) & 0,5 & 0,81 \\
\hline Universidade Federal de Viçosa (UFV) & 0,5 & 0,81 \\
\hline Universidade Federal do Estado do Rio de Janeiro (UNIRIO) & 0,5 & 0,81 \\
\hline Centro de Tecnologia da Informação Renato Archer & 0,33 & 0,53 \\
\hline Faculdade Governador Ozanam Coelho (FAGOC) & 0,33 & 0,53 \\
\hline $\begin{array}{l}\text { Instituto Federal de Educação, Ciência e Tecnologia Sul Rio } \\
\text { Grandense (IFSul-Passo Fundo) }\end{array}$ & 0,33 & 0,53 \\
\hline Universidade Federal de Alfenas (UNIFAL) & 0,33 & 0,53 \\
\hline Universidade Federal do ABC (UFABC) & 0,33 & 0,53 \\
\hline
\end{tabular}




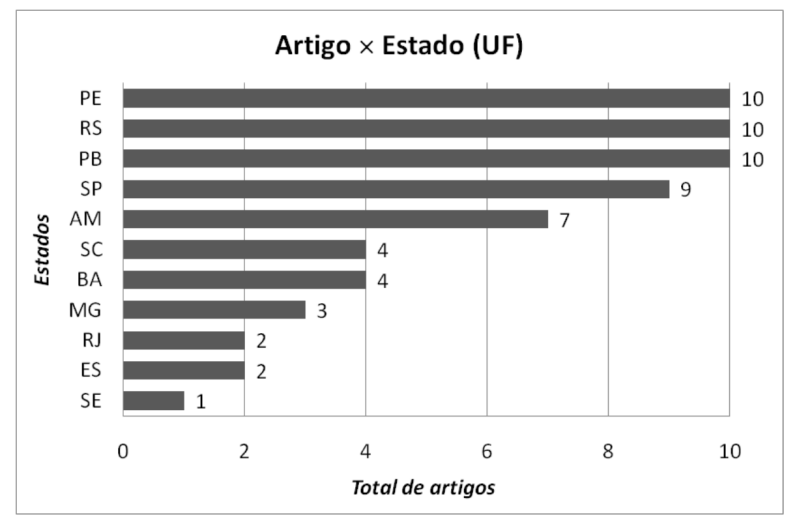

Figura 4: Total de artigos por estado (UF).

\section{Considerações Finais}

Com base no levantamento apresentado, é possível perceber que nos últimos anos tem aumentado o interesse pelo ensino de fundamentos da computação no contexto escolar brasileiro. Entrementes, todos versam o PC objetivando sempre a desenvolver o raciocínio computacional e lógico em crianças e adolescentes.

Este trabalho, fez um levantamento dos últimos 6 anos, de 2010 a 2015, em 7 principais eventos nacionais na área de Informática na Educação. Os primeiros indícios da presença do PC foram encontrados em Anais de eventos a partir de 2010, onde a cada ano posterior, pôde-se perceber um considerável aumento do número de projetos envolvendo o PC.

Conforme a análise dos dados levantados, é possível observar que a maioria dos projetos trabalham com a abordagem Algoritmos e Programação, seguida da abordagem Desplugada, esta última envolvendo diversos conceitos da Computação, e na sua grande maioria, tendo como referência as atividades do livro Computer Science Unplugged. Além disso, as ferramentas que têm sido adotadas na estratégia de AeP parecem demonstrar uma tendência para um ensino de programação por meio do "montar/encaixar comandos" ao invés de "codificar/escrever linhas de código".

Dentre os aspectos considerados nesta pesquisa, identificou-se pouco incentivo ao trabalho colaborativo e/ou interdisciplinar. A grande maioria, independente da abordagem, seja ela Algoritmos e Programação, Robótica, Jogos, Desplugada, entre outras, trabalham apenas os conceitos e conteúdos da ciência da computação, e poucos integram outras disciplinas que fazem parte do currículo básico escolar. A colaboração, também é outro aspecto pouco explorado, sendo abordada pela maioria dos trabalhos apenas como execução de tarefas em 
grupos.

Para os projetos que ainda estão em execução de suas atividades, a maioria tem como proposta para o futuro prosseguir aplicando o PC nas escolas, visando aumentar o número de turmas e de alunos, bem como desenvolver novas metodologias para aplicação destas atividades, de forma que as habilidades do PC que estão sendo desenvolvidas fiquem o mais próximo possível da realidade dos alunos.

Em crescimento no Brasil, espera-se que a aplicação desta nova metodologia de ensino se popularize e se expanda em um futuro próximo. Como projetos futuros, pretende-se ampliar este levantamento para além da Educação Básica, e considerando as pesquisas em desenvolvimento no exterior.

\section{Agradecimentos}

Os autores agradecem o apoio financeiro recebido do MEC/SESu (Programa PROEXT).

\section{Contribuição dos autores:}

- Adriana Bordini, Christiano Martino Otero Avila, Yuri Rocha Weisshahn e Mônica Marques da Cunha: conduziram a pesquisa e a escrita do manuscrito.

- Simone André da Costa Cavalheiro: coordenou a elaboração do trabalho, supervisionou a realização da pesquisa e direcionou a redação do texto.

- Luciana Foss: auxiliou na redação do texto e realizou correções.

- Marilton Sanchotene Aguiar e Renata Hax Sander Reiser: revisaram o texto do trabalho.

\section{Referências}

[1] J. M. Wing, “Computational thinking," Communications of the ACM, vol. 49, pp. 33-35, Mar. 2006.

[2] CSTA, "Computational thinking - leadership toolkit," 2011.

[3] Y. Weisshahn, A. I. R. Duarte, S. A. d. C. Cavalheiro, L. Foss, M. Aguiar, A. Du Bois, A. M. P. Fleischmann, R. H. S. Reiser, and C. F. d. B. Piana, "Pensamento computacional no Brasil: O estado da arte," in Anais do Workshop Escola de Informática Teórica, 2015 . 
[4] F. Arantes, T. Amiel, and G. Fedel, "Nos rumos da autonomia tecnológica-desafios e lições aprendidas para a formação de jovens," in XX Anais do Workshop de Informática na Escola, 2014.

[5] R. d. França and H. d. Amaral, "Proposta metodológica de ensino e avaliação para o desenvolvimento do pensamento computacional com o uso do scratch," in XX Anais do Workshop de Informática na Escola, 2013.

[6] Á. Lima and D. Sousa, "Experiência no programa institucional de bolsas de iniciação à docência (pibid): Desenvolvimento do raciocínio lógico e algoritmo na educação básica," in IV Anais dos Workshops do Congresso Brasileiro de Informática na Educação, 2015 .

[7] Á. Lima and D. d. Sousa, "Desenvolvimento do raciocínio lógico e algoritmo através do programa institucional de bolsas de iniciação à docência no ensino fundamental," in IV Anais dos Workshops do Congresso Brasileiro de Informática na Educação, 2015.

[8] E. Z. d. A. Machado, I. R. Vasconcelos, and K. Malta, "Uma experiência em escolas de ensino médio e fundamental para a descoberta de jovens talentos em computação," in XVIII Anais do Workshop sobre Educação em Computação, 2010.

[9] L. F. Meneses, L. F. F. Mai, E. d. Rosario, J.; Oliveira, and R. Gomes, "Introcomp: Atraindo alunos do ensino médio para uma instigante experiência com a programação," in XXIII Anais do Workshop sobre Educação em Computação, 2015.

[10] N. Ramos, C. Freitas, S. Avila, P. D. P. Costa, V. Testoni, and J. F. Borin, "Ensino de programação para alunas de ensino médio: Relato de uma experiência," in XXIII Anais do Workshop sobre Educação em Computação, 2015.

[11] R. d. S. Rodrigues, L. A. d. M. Morais, S. S. Dantas, J. G. Lopes Filho, C. R. G. I. Abilio, and P. R. Suárez, "Ensino de algoritmos e linguagem de programação no nível médio: Um relato de experiência," in XXI Anais do Workshop sobre Educação em Computação, 2013.

[12] A. Sousa, S. Silva, A. A. Raiol, J. Sarges, and F. Bezerra, "O universo ludico da programação de computadores com logo no ensino fundamental," in XXIII Anais do Workshop sobre Educação em Computação, 2015.

[13] D. F. d. Sousa, "Desenvolvendo a lógica e algoritmos no ensino médio," in XIX Anais do Workshop de Informática na Escola, 2013.

[14] C. Ferreira, F. Gonzaga, and R. Santos, "Um estudo sobre a aprendizagem de lógica de programação utilizando programação por demonstração," in XVIII Anais do Workshop sobre Educação em Computação, 2010. 
[15] P. D. Scaico, A. A. Lima, J. B. B. d. Silva, S. Azevedo, L. F. Paiva, E. H. S. Raposo, Y. Alencar, and J. P. Mendes, "Programação no ensino médio: Uma abordagem de ensino orientado ao design com scratch," in XVIII Anais do Workshop de Informática na Escola, 2012.

[16] P. D. Scaico, E. F. Corlett, L. F. Paiva, E. H. S. Raposo, and Y. Alencar, "Relato da utilização de uma metodologia de trabalho para o ensino de ciência da computação no ensino médio," in XVIII Anais do Workshop de Informática na Escola, 2012.

[17] W. F. Gomes, C. S. Louzada, M. A. S. N. Nunes, E. M. Salgueiro, and B. T. Andrade, "Incentivando meninas do ensino médio à área de ciência da computação usando o scratch como ferramenta," in Anais do Workshop de Informática na Escola, 2014.

[18] N. Oro, A. Pazinato, A. Teixeira, and Á. Gross, "Olimpíada de programação de computadores para estudantes do ensino fundamental: A interdisciplinaridade por meio do software scratch,” in Anais do Workshop de Informática na Escola, 2015.

[19] A. Teixeira, J. R. Martins, F. Batistela, A. Pazinato, and N. Oro, "Programação de computadores para alunos do ensino fundamental: A escola de hackers," in Anais do Workshop de Informática na Escola, 2015.

[20] A. C. T. d. Santos, J. A. Monteiro, P. R. B. Machado, K. C. T. Lins, T. d. A. R. Ramos, and L. V. Batista, "Ensino de programação para olimpíada brasileira de informática," in Anais do Workshop de Informática na Escola, 2015.

[21] F. Viel, A. Raabe, and C. Zeferino, "Introdução à programação e à implementação de processadores por estudantes do ensino médio," in XX Anais do Workshop de Informática na Escola, 2014.

[22] C. G. v. Wangenheim, V. R. Nunes, and G. D. d. Santos, "Ensino de computação com scratch no ensino fundamental-um estudo de caso," Revista Brasileira de Informática na Educação, 2014.

[23] P. D. Scaico, A. A. d. Lima, S. Azevedo, J. B. B. d. Silva, E. H. Raposo, Y. Alencar, J. P. Mendes, A. Scaico, and L. F. Paiva, "Ensino de programação no ensino médio: Uma abordagem orientada ao design com a linguagem scratch," in Revista Brasileira de Informática na Educação, 2013.

[24] C. Torezani, L. B. d. C. Chagas, and O. d. L. Tavares, "Newprog-um ambiente online para crianças aprenderem programação de computadores," in XIX Anais do Workshop de Informática na Escola, 2013.

[25] A. L. S. O. d. Araújo, P. D. Scaico, L. F. d. Paiva, H. d. M. Rabêlo, L. d. L. Santos, F. I. R. Pessoa, J. M. Targino, and L. d. S. Costa, "Aplicação da taxonomia de bloom 
no ensino de programação com scratch," in XIX Anais do Workshop de Informática na Escola, 2013.

[26] D. R. Krathwohl, “A revision of bloom's taxonomy: An overview," Theory into practice, vol. 41, no. 4, pp. 212-218, 2002.

[27] N. S. Bastos, D. F. Adamatti, and F. A. H. Carvalho, "Ensino de lógica de programação no ensino médio e suas implicações na neurociências," in Anais do Simpósio Brasileiro de Informática na Educação, 2015.

[28] R. d. França, W. d. Silva, and H. d. Amaral, "Ensino de ciência da computaçao na educaçao básica: Experiências, desafios e possibilidades," in XX Workshop sobre Educação em Computação, 2012.

[29] F. Bezerra and K. Dias, "Programaç ao de computadores no ensino fundamental: Experiências com logo e scratch em escola pública," in XXII Anais do Workshop sobre Educação em Computação, 2014.

[30] G. d. O. Mattos, D. R. D. d. Silva, and J. A. Moreira, "A utilização de kits de robótica como ferramenta para o ensino de programação à meninas do ensino médio," in XXIII Anais do Workshop sobre Educação em Computação, 2015.

[31] L. R. Amaral, G. B. e. Silva, and E. Pantaleão, "Plataforma robocode como ferramenta lúdica de ensino de programação de computadores-pesquisa e extensão universitária em escolas públicas de minas gerais," in Anais do Simpósio Brasileiro de Informática na Educação, 2015.

[32] J. V. V. D’Abreu, L. G. B. Mirisola, and J. J. G. Ramos, “Ambiente de robótica pedagógica com br_gogo e computadores de baixo custo: Uma contribuição para o ensino médio," in Anais do Simpósio Brasileiro de Informática na Educação, 2011.

[33] T. S. C. d. Silva, A. S. C. d. Silva, and J. C. B. d. Melo, "Ensino de algoritmos a nível médio utilizando música e robótica: Uma abordagem lúdica," in Workshop sobre Educação em Computação, 2011.

[34] F. d. C. Barbosa, M. L. Alexandre, D. B. Alves, D. C. d. Menezes, G. L. Campos, Y. Nakamura, A. J. d. S. Junior, and C. R. Lopes, "Robótica educacional em prol do ensino de matemática," in Anais do Workshop de Informática na Escola, 2015.

[35] P. Schoeffel, P. Moser, G. M. Varela, L. R. Durigon, G. C. d. Albuquerque, and M. d. S. Niquelati, "Uma experiência no ensino de pensamento computacional e fomento à participação na olimpíada brasileira de informática com alunos do ensino fundamental," in Workshop de Ensino em Pensamento Computacional, Algoritmos e Programação, 2015. 
[36] M. S. Pereira, B. H. Reis, G. B. Oliveira, G. E. Farias, A. J. S. Júnior, and C. R. Lopes, "Usando enchanting em robótica educativa," in Anais do Workshop de Informática na Escola, 2014.

[37] Y. Q. d. Aguiar, B. K. Maciel, S. D. G. Mattos, L. B. Soares, and V. M. d. Oliveira, "Introdução à robótica e estímulo à lógica de programação no ensino básico utilizando

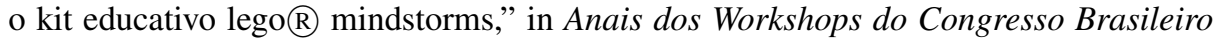
de Informática na Educação, 2015.

[38] C. S. Pimentel, K. Revoredo, and F. Sampaio, "Mecatrônica educacional apoiando o aprendizado de conceitos de física e matemática: Um estudo de caso," in Anais do Workshop de Informática na Escola, 2015.

[39] J. V. V. D'Abreu and B. L. Bastos, "Robótica pedagógica e currículo do ensino fundamental: Atuação em uma escola municipal do projeto uca," in Revista Brasileira de Informática na Educação, 2015.

[40] A. S. Charão, P. P. d. A. Barcelos, B. R. d. Azevedo, and J. C. D. Lima, "Clube de computação para alunos de ensino médio: um relato de experiência," in Workshop sobre Educação em Computação, 2015.

[41] S. M. Souza, M. d. S. Rios, C. A. Rodrigues, D. M. B. d. Santos, and R. A. Bittencourt, "Oficinas de programação com ambientes ludicos para meninas do ensino fundamental," in Anais do XXI Workshop sobre Educação em Computação, 2015.

[42] W. P. Batista, H. S. Chalegre, J. P. S. Sena, A. H. Santos, D. M. B. dos Santos, C. A. Rodrigues, and R. A. Bittencourt, "Oficinas de aprendizagem de programação em uma escola pública através do ambiente scratch," in XXII Anais do Workshop sobre Educação em Computação, 2015.

[43] F. Ramos and L. d. S. Teixeira, "Significação da aprendizagem através do pensamento computacional no ensino médio: uma experiência com scratch," in Anais do Workshop de Informática na Escola, 2015.

[44] S. F. Siva, A. F. Barbosa, A. A. Souza, E. G. d. Silva, M. L. S. d. Oliveira, S. R. d. Silva Neto, and W. O. Santos, "Relato de experiência de ensino de computação no ensino fundamental em estágio supervisionado da universidade de pernambuco no campus garanhuns," 2015.

[45] T. P. Falcão and R. Barbosa, “"Aperta o Play!"análise da interação exploratória em um jogo baseado em pensamento computacional," in Anais do Simpósio Brasileiro de Informática na Educação, 2015. 
[46] D. L. Marques, L. F. S. Costa, M. A. d. A. Silva, and A. D. D. S. Rebouças, "Atraindo alunos do ensino médio para a computação: Uma experiência prática de introdução a programação utilizando jogos e python," in Anais do Workshop de Informática na Escola, 2011.

[47] R. S. de França and P. Tedesco, "Explorando o pensamento computacional no ensino médio: do design à avaliação de jogos digitais," in Anais dos Workshops do Congresso Brasileiro de Informática na Educação, 2015.

[48] R. França and P. Tedesco, "Um modelo para a aprendizagem do pensamento computacional aliado à autorregulação," in Anais do Concurso de TCC, Dissertações e Teses do Congresso Brasileiro de Informática na Educação, 2015.

[49] A. Raabe, G. Zanchett, and A. Vahldick, "Jogos de programar como uma abordagem para os primeiros contatos dos estudantes com à programação," in Anais dos Workshops do Congresso Brasileiro de Informática na Educação, 2015.

[50] C. Shimohara and E. Sobreira, "Criando jogos digitais para a aprendizagem de matemática no ensino fundamental I," in Anais do Workshop de Informática na Escola, 2015.

[51] C. Rodriguez, A. M. Zem-Lopes, L. Marques, and S. Isotani, "Pensamento computacional: transformando ideias em jogos digitais usando o scratch," in Anais do Workshop de Informática na Escola, 2015.

[52] A. Vieira, O. Passos, and R. Barreto, "Um relato de experiência do uso da técnica computação desplugada," in Anais do Workshop sobre Educação em Computação, 2013.

[53] T. Costa, A. Batista, M. Maia, L. Almeida, and A. Farias, "Trabalhando fundamentos de computação no nível fundamental: experiência de licenciandos em computação da universidade federal da paraíba," in XX Workshop de Educação em Computação, 2012.

[54] G. M. d. Campos, S. Cavalheiro, L. Foss, A. M. Pernas, C. F. d. B. Piana, M. Aguiar, A. Du Bois, and R. Reiser, "Organização de informações via pensamento computacional: Relato de atividade aplicada no ensino fundamental," in Anais do Workshop de Informática na Escola, 2014.

[55] M. L. S. Oliveira, A. A. d. Souza, A. F. Barbosa, and E. F. S. Barreiros, "Ensino de lógica de programação no ensino fundamental utilizando o scratch: um relato de experiência," in XXII Workshop sobre Educação em Computação, 2014.

[56] F. Bezerra, "Bem mais que os bits da computação desplugada," in Anais do Workshop de Informática na Escola, 2014. 
[57] A. V. d. S. Barbosa, A. F. Pereira Neto, R. N. R. d. Oliveira, T. L. S. d. Costa, A. L. S. Araújo, and F. V. S. Costa, "O ensino de conceitos computacionais para alunos do ensino médio: relato de experiência de uma gincana e das estratégias utilizadas pelos alunos na resolução das atividades desplugadas," in Workshop sobre Educação em Computação, 2015 .

[58] D. d. C. Araújo, A. N. Rodrigues, C. V. d. A. Silva, and L. S. Soares, "O ensino de computação na educação básica apoiado por problemas: Práticas de licenciandos em computação," in Workshop sobre Educação em Computação, 2015.

[59] L. F. d. Paiva, A. C. Ferreira, C. Rocha, J. Barreto, A. Melhor, R. Lopes, and E. Matos, "Uma experiência piloto de integração curricular do raciocínio computacional na educação básica," in do Workshop de Ensino em Pensamento Computacional, Algoritmos e Programação, 2015.

[60] T. Bell, I. H. Witten, and M. Fellows, Computer Science Unplugged: Off-line activities and games for all ages. Citeseer, 1998.

[61] L. Hinterholz and M. K. d. Cruz, "Desenvolvimento do pensamento computacional: um relato de atividade junto ao ensino médio, através do estágio supervisionado em computação iii," in Anais do Workshop de Informática na Escola, 2015.

[62] G. Santos, P. Finkenauer, B. Arnhold, S. A. d. C. Cavalheiro, C. Piana, L. Foss, M. Aguiar, A. Pernas, A. Du Bois, and R. Reiser, "Relato sobre o ensino de algoritmos a estudantes do quinto ano do ensino público fundamental," in Workshop Escola de Informática Teórica, 2015.

[63] D. Andrade, T. Carvalho, J. Silveira, S. Cavalheiro, L. Foss, A. M. Fleischmann, M. Aguiar, and R. Reiser, "Proposta de atividades para o desenvolvimento do pensamento computacional no ensino fundamental," in Anais do Workshop de Informática na Escola, 2013.

[64] F. L. Arantes and J. M. L. d. S. Ferreira, "Uma dinâmica para ensino de conceitos fundamentais de programação," in Anais do Workshop de Ensino em Pensamento Computacional, Algoritmos e Programação, 2015.

[65] G. Santos, W. Silva, S. Cavalheiro, L. Foss, M. Aguiar, A. M. Pernas, A. Du Bois, and R. Reiser, "Proposta de atividade para o quinto ano do ensino fundamental: Algoritmos desplugados," in Anais do Workshop de Informática na Escola, 2015.

[66] D. R. Garrison and H. Kanuka, "Blended learning: Uncovering its transformative potential in higher education,” vol. 7, no. 2, pp. 95-105, 2004. 
[67] A. C. Ferreira, , A. Melhor, J. d. S. Barreto, L. F. d. Paiva, and E. Matos, "Experiência prática interdisciplinar do raciocínio computacional em atividades de computação desplugada na educação básica," in Anais do Workshop de Informática na Escola, 2015.

[68] T. C. S. Gomes and J. C. B. d. Melo, "O pensamento computacional no ensino médio: Uma abordagem blended-learning," in Anais do XXI Workshop sobre Educação em Computação, 2013. 